\title{
Improving smoking cessation policy by assessing user demand for an inpatient smoking cessation service in adult psychiatric wards
}

\author{
Kathy Liu, Andrew Creamer \\ South London and Maudsley NHS Foundation Trust, UK
}

\begin{abstract}
Smoking rates are higher among people with mental health conditions compared to the general population. Smoking reduces physical, mental and financial well-being, and interacts with psychotropic drugs. An inpatient admission provides an opportunity to engage and support smokers in smoking cessation. Compliance with Trust/NICE smoking cessation guidelines was assessed in two inpatient wards, and a questionnaire evaluated user demand for an inpatient smoking cessation service. A need for improved documentation of smoking status to identify and treat smokers routinely was revealed.

A new electronic health form was designed and introduced, and a clear pathway for onward referrals was developed. This intervention preceded the introduction of the Trust-wide smoke free policy from October 2014. The intervention doubled rates of documentation of smoking status, cessation advice and offer of NRT/referral. There were large differences between the two wards, highlighting the need for a tailored approach.
\end{abstract}

\section{Problem}

The documentation of smoking status and referral pathway to smoking cessation services is unclear for inpatients in a large psychiatric teaching hospital. NICE guidelines [1] and the South London and Maudsley NHS Trust smoking policy [2] have highlighted the importance of identifying smokers, encouraging cessation, and assessing for appropriate referral to specialist support. With the relatively long duration of most psychiatric admissions, an inpatient admission provides an excellent opportunity to engage service users in education about the harmful effects of smoking and to encourage and support cessation.

However, from our experiences on the wards this appears to be an aspect of care that is not prioritised. It is difficult to document current smoking status clearly in the electronic patient records, staff attitudes can hinder smoking cessation processes (smoking seen as a "reward" for patients), and no clear referral pathway currently exists for patients who do express a wish to stop smoking.

\section{Background}

Smoking rates are higher among people with mental health conditions compared to the general population. This contributes to the higher rates of physical co-morbidity and shorter life expectancy in this population. Furthermore, smoking has an associated financial burden, and can interact with the pharmacokinetics of psychotropic drugs such as clozapine, meaning higher doses are required. Smoking cessation has been shown to improve mental health and quality of life in a recent meta-analysis.[4] This is contrary to widely-held views that intervening with smokers might be detrimental to their mental health. In the context of a national push to make many hospital sites smoke-free, an inpatient admission to a psychiatric ward should equally provide an opportunity to engage and support smokers in smoking cessation.

NICE guidelines recognise the importance of documenting the current smoking status of patients, and encouraging and supporting cessation by healthcare professionals.[1] Recent published guidelines within our Trust further reinforce this.[2] The guidelines advise that all smokers be assessed for motivation to stop smoking, and those expressing a desire to stop should be referred to level 2 specialist cessation services.

A recently published quality improvement report [5] has encountered a similar problem in a general medical inpatient setting, but to our knowledge this is the first published smoking cessation audit report on a psychiatric inpatient population.

\section{Baseline measurement}

The initial cycle was performed as a snapshot audit of all current patients on two acute male psychosis wards. The study cohort was comprised of 40 male patients, average age 45 , with a mean admission duration of 56 days. Eighteen (45\%) were current smokers.

Data were collected by two methods. Firstly, patient electronic records were analysed to assess compliance with documentation of smoking status and desire to stop and whether any intervention (advice, nicotine replacement therapy, or referral) had been made. Secondly, a questionnaire was given to patients that assessed smoking history, motivation to stop, and whether they preferred to access cessation support as inpatient or outpatient.

From patient electronic records, 15\% (six) had smoking status documented during the current admission and all of these had a 
documented discussion advising them to stop. Seventy percent of patients had smoking status documented at least once in last five years. The documentation of smoking status was part of a larger electronic record form and did not always capture useful information, for example, the options were either "Yes" "No" or "Unsure" for most of the questions. It did not facilitate subsequent referral to a cessation service.

Sixteen of the 18 self-reported smokers completed the questionnaire. Of these, seven (44\%) reported motivation to quit, but only one of these had smoking status documented during admission. None of these motivated smokers had been offered a referral to a specialist service, and $86 \%$ stated they would prefer an inpatient cessation service.

\section{Design}

Our results indicated a need to facilitate the useful documentation of smoking status and also for developing a clear referral pathway for patients expressing a wish to stop. Since all documentation in the Trust is via electronic records, a new smoking cessation and referral form was designed and launched on the electronic system in June 2014. Smoke free policy stakeholders were consulted to agree on the content. The form provided a clear place to document current smoking information including smoking status, quantity smoked per day, and whether intervention - advice to stop, nicotinereplacement therapy, and referral - was offered.

To create a viable referral pathway, we liaised with the specialist smoking cessation clinic currently operating in the Trust, previously only utilised by outpatients. After discussing our baseline results, they agreed to accept direct referrals from inpatients via the new online form.

\section{Strategy}

PDSA cycle 1: The electronic form was designed in collaboration with the professor of addictions in the Trust, smoke free policy stakeholders, as well as the lead nurse on both wards for feedback on content and ease of use. An example of a modification made at this stage was one question that asked the time to first cigarette. While this was useful from a research point of view, was clinically less relevant and might compromise the practicality and succintness of the form. This was agreed and the form was adjusted accordingly.

PDSA cycle 2: After a redesign the form was rolled out on the two inpatient psychiatric wards after education sessions with the ward staff were undertaken about the pilot intervention. The phone number for referral to a specialist clinic was included in the form. The results after one month were positive and overall rates of documentation had doubled from $15 \%$ to $36 \%$, however one ward achieved $72 \%$ and the other ward $0 \%$. Other feedback received included that sometimes the telephone line may be busy and this impaired the completion of the form, or was confusing as to whether it had been followed up.
PDSA cycle 3: Meeting with smoke free policy stakeholders to discuss reasons behind these differences between wards. It was determined that one ward had dedicated smoking cessation trained nurses who were taking ownership of the assessment and management of patient smoking status. To help support the ward which did not have the dedicated trained nurses, the electronic form was linked to a live referral system to a specialist smoking cessation clinic, so referrals could be made directly by submitting the form instead of waiting at the end of a phone line. These changes were made in time for the Trust wide smoke free policy implementation.

PDSA cycle 4: Another snapshot measurement of rates of smoking form completion on the same two wards was performed three months after implementation of the Trust wide smoke free policy. Rates of completion were actually lower and one ward still had lower rates than the other, $30 \%$ compared to $0 \%$. However, as the smoke free policy was Trust wide, three additional wards were selected randomly and rates of completion were measured. Ward 3 had $55 \%$ completion rates, and of the five documented smokers on the ward one accepted a referral to smoking cessation clinic. Ward 4 had $30 \%$ completion rates and of five documented smokers, one accepted a referral to smoking cessation clinic. Ward 6 had $83 \%$ completion rates with all documented smokers declining a referral. Reasons behind the differences will be investigated by the team.

\section{Results}

Results following PDSA cycle 1:

The same snapshot methodology was used as in the baseline audit, with the study population drawn from the same two wards.

The study cohort consisted of 36 male patients with a mean admission duration of 55.8 days.

$36 \%$ (13) had a smokers screening form completed during the current admission. All of these patients had smoking status documented, were advised to quit, offered nicotine replacement therapy and a referral to smoking cessation clinic.

However, the results showed a significant discrepancies between the two wards, with ward 1 had $0 \%$ completion rates, Ward 2 had $72 \%$ completion rates.

Results following PDSA Cycle 3:

The same snapshot methodology was used as in the baseline audit, with the study population drawn from the same two wards. Rates of completion were actually lower and one ward still had lower rates than the other, $30 \%$ compared to $0 \%$.

However, as the smoke free policy was Trust wide, three additional wards were selected randomly and rates of completion were measured. Ward 3 had $55 \%$ completion rates, and of the five documented smokers on the ward one accepted a referral to smoking cessation clinic. Ward 4 had $30 \%$ completion rates and of 5 documented smokers one accepted a referral to smoking 


\section{BMJ Quality Improvement Reports}

cessation clinic. Ward 6 had $83 \%$ completion rates with all documented smokers declining a referral.

\section{Lessons and limitations}

This project introduced an intervention which demonstrably improved the service the Trust provides to patients who smoke. Several lessons were learned over the course of this project. Firstly, when designing a new intervention, the wide range of healthcare professionals that could be affected should be considered, including possible reasons for resistance. We noted that some of the healthcare professionals were smokers themselves, so effective communication and education was essential for getting everyone on board. Similarly, communication with a variety of stakeholders was essential for implementing the intervention. We worked with a range of staff at the Trust, including ward nurses and managers, the IT department, and addiction specialists. Listening to the views and opinions of all involved was essential for the successful implementation of the project.

One of the limitations of our study was a relatively small sample size. Additionally we were involved with the training of professionals on the two wards initially measured; we were not involved with further smoking cessation training that occurred Trust-wide involving the additional Wards 3,4 , and 5 . The variability between wards is interesting and could be down to several factors. One issue we noted was that the original Physical Health screening form with some smoking questions was still in existence, which could be a source repetition and confusion about the use of our smoking cessation form. Secondly, the Physical Health form is associated with financial targets whilst the smoking cessation form is still in a pilot stage. We will aim to work towards replacing the smoking section of the physical health form with a specialised smoking cessation form to reduce unhelpful duplication.

\section{Conclusion}

The intervention improved rates of documentation of smoking status, cessation advice, and offer of nicotine replacement therapy and referral for inpatients on two psychiatric wards. Differences between wards highlight disparities in attitude and training between health professionals and the need for a tailored approach for implementation.

\section{References}

1. NICE Clinical guidelines PH48: Smoking Cessation in secondary care: acute, maternity and mental health services (November 2013).

2. South London and the Maudsley NHS Foundation Trust Policy - Smoke Free Policy, June 2012.

3. West R, McNeill A, Raw M Smoking Cessation Guidelines for Health Professionals - an update. Thorax 2000;55:987-99.

4. Taylor G, McNeill A, Girling A, Farley A, Lindson-Hawley N, Aveyard $P$ et al. Change in mental health after smoking cessation: systematic review and meta-analysis BMJ 2014; 348:g1151.

5. Bickerstaffe G. Smoking cessation for hospital inpatients BMJ Qual Improv Report 2014; 3.

\section{Declaration of interests}

Nothing to declare.

\section{Acknowledgements}

Professor Ann McNeill, Debbie Robson. 